\title{
A large class of new bivariate copulas and their properties
}

\section{Zahra Sharifonnasabi, Mohammad Hossein Alamatsaz ${ }^{1}$ and Iraj Kazemi University of Isfahan}

\begin{abstract}
In this paper, we shall construct a large class of new bivariate copulas. This class happens to contain several known classes of copulas, such as Farlie-Gumbel-Morgenstern, Ali-Mikhail-Haq and Barnett-Gumbel, as its especial members. It is shown that the proposed copulas improve the range of values of correlation coefficient and thus they are more applicable in data modeling. We shall also reveal that the dependent properties of the base copula are preserved by the generated copula under certain conditions. Several members of the new class are introduced as instances and their range of correlation coefficients are computed.
\end{abstract}

\section{Introduction}

Copulas are useful tools in constructing joint distributions. In fact, a copula is a multidimensional distribution function (d.f.) whose marginals are uniformly distributed on $[0,1]$. We shall only focus on bivariate copulas and their properties in this manuscript.

A bivariate copula is a function $C: I^{2} \rightarrow I(=[0,1])$ which satisfies the boundary conditions

$$
C(t, 0)=C(0, t)=0 \quad \text { and } \quad C(t, 1)=C(1, t)=t, \quad t \in I
$$

and the 2-increasing condition

$$
C\left(u_{2}, v_{2}\right)-C\left(u_{1}, v_{2}\right)-C\left(u_{2}, v_{1}\right)+C\left(u_{1}, v_{1}\right) \geq 0
$$

for all $u_{1}, u_{2}, v_{1}, v_{2}$ in $[0,1]$ such that $u_{1} \leq u_{2}$ and $v_{1} \leq v_{2}$.

According to Sklar' s theorem (see Sklar (1959)), using a two-dimensional copula $C(\cdot, \cdot)$, any bivariate d.f. $H_{X, Y}$ can be represented as a function of its marginal distributions $F_{X}$ and $F_{Y}$. In fact, if $F_{X}$ and $F_{Y}$ are continuous d.f.'s, then there exists a unique copula such that

$$
H_{X, Y}(x, y)=C\left(F_{X}(x), F_{Y}(y)\right),
$$

where

$$
C(u, v)=P(U \leq u, V \leq v), \quad U \equiv F_{X}(x), \quad V \equiv F_{Y}(y) .
$$

\footnotetext{
${ }^{1}$ Corresponding author.

Key words and phrases. FGM copulas, stochastic dependence, tail dependence, Spearman's $\rho$. Received June 2015; accepted January 2017.
} 
Assuming that $C$ is differentiable with respect to its both arguments, equation (1.2) is satisfied if

$$
c(u, v) \equiv \frac{\partial^{2} C(u, v)}{\partial u \partial v} \geq 0 .
$$

Furthermore, it is known that

$$
\begin{aligned}
& \frac{\partial C(u, v)}{\partial u}=P(V \leq v \mid U=u) \geq 0, \\
& \frac{\partial C(u, v)}{\partial v}=P(U \leq u \mid V=v) \geq 0 .
\end{aligned}
$$

For further details about copulas, see Nelsen (2006).

A known and useful family of copulas is FGM family. This family is used, e.g., for competing risk problems Tolley and Norman (1979), the joint occurrence of certain trace elements in water Cook and Johnson (1986) and a robustness issue Delahorra and Fernandez (1995). However, a shortcoming of FGM copulas is that they do not allow modeling of high dependencies because their spearman's $\rho$ is limited to $\left[\frac{-1}{3}, \frac{1}{3}\right]$. To overcome this problem, many authors such as Huang and Kotz (1999), Bairamov and Kotz (2002), Amblard and Girard (2002, 2009), Amini, Jabbari and Mohtashami Borzadaran (2011) have tried to extend this family. Recently Klein and Christa (2011) proposed a class of copulas which contains FGM copulas as follows: A bivariate function

$$
C(u, v ; \theta, \gamma)=u v[1+\theta \phi(u) \phi(v)]^{\frac{1}{\gamma}} \quad \forall u, v \in I
$$

is a copula if the function $\phi:[0,1] \rightarrow \mathfrak{R}$ is a differentiable and monotone function satisfying

1. $\phi(1)=0$,

2. $|\phi(u)| \leq 1, u \in[0,1]$,

3. $D=\left\{\gamma \neq 0 ;\left|\phi(u)+\frac{u \phi^{\prime}(u)}{\gamma}\right| \leq 1, u \in[0,1]\right\}$,

4. either $\gamma \in D$ and $\theta \in[-1,1]$ such that $\theta \gamma>0$, or $\phi$ is nonnegative and monotonely decreasing or nonpositive and monotonely increasing on $u \in[0,1]$ and

$$
\gamma \in D \cap(0,1] \text { and } \theta \in[-1,0)
$$

or

$$
\gamma \in D \cap(-\infty,-1] \text { and } \theta \in(0,1]
$$

Also, the bivariate function

$$
C(u, v ; \theta)=u v e^{\theta \phi(u) \phi(v)}, \quad u, v \in I
$$

is a copula for $\theta \in[0,1]$ if

1. $\phi(1)=0$, 
2. $|\phi(u)| \leq 1, u \in[0,1]$,

where $\phi:[0,1] \rightarrow \Re$ is a differentiable and monotone function, and it is a copula for $\theta \in[-1,0]$ if

1. $\phi(1)=0$,

2. $\left|u \phi^{\prime}(u)\right| \leq 1, u \in[0,1]$,

where $\phi:[0,1] \rightarrow \mathfrak{R}$ is differentiable, nonnegative and monotonely decreasing or nonpositive and monotonely increasing on $[0,1]$.

The remainder of the paper is organized as follows. In Section 2, replacing the independent copula $u v$ in (1.7) and (1.8) by a general copula $K(u, v)$, we shall introduce a new class of copulas which extends several known classes of copulas, such as FGM, AMH and BG and several other families. We shall discuss conditions under which our generalization is justified. Several illustrating examples are provided in Section 3. Dependent properties of the new class are studied in Section 4. In Section 5, we shall discuss tail dependencies of members of this new family. Measures of association are discussed in Section 6, where it is observed that the new family can be used to model data with a wider range of correlation than the FGM family.

\section{A new class of bivariate copulas}

Consider the bivariate copula (1.7) and (1.8) of Klein and Christa (2011). We replace the coefficient $u v$, the independent copula, by an arbitrary copula $K(u, v)$. That is, we consider the bivariate function

$$
C(u, v ; \theta, \gamma)=K(u, v)[1+\theta \phi(u) \phi(v)]^{\frac{1}{\gamma}} \quad \forall u, v \in I,
$$

where $\theta \in[-1,1]$ and $\gamma \in \Re /\{0\}$ and

$$
C(u, v ; \theta)=K(u, v) e^{\theta \phi(u) \phi(v)} \quad \forall u, v \in I,
$$

where $\theta \in[-1,1]$.

The next theorems provide certain conditions under which $C(u, v ; \gamma, \theta)$ and $C(u, v ; \theta)$ in (2.1) and (2.2), respectively, are indeed well defined copulas. First, in order to simplify the statements of our theorems, we set up the following assumptions and definitions:

Assumptions:

1. $\frac{\partial^{2}}{\partial u \partial v} \ln K(u, v) \geq 0, \forall(u, v) \in I^{2}$.

2. $G=\left\{\gamma \neq 0 ;\left|\phi(u)+\frac{\phi^{\prime}(u)}{\gamma \frac{\partial}{\partial u} \ln K(u, v)}\right| \leq 1,\left|\phi(v)+\frac{\phi^{\prime}(v)}{\gamma \frac{\partial}{\partial v} \ln K(u, v)}\right| \leq 1,(u, v) \in I^{2}\right\}$.

3. $0 \leq \phi(u) \leq 1$ and be decreasing or $-1 \leq \phi(u) \leq 0$ and be increasing.

4. $\phi(u)$ is a negative and nondecreasing function and

$$
\phi^{\prime}(u) \leq \frac{\partial}{\partial u} \ln K(u, v), \quad \forall v \in I .
$$


5. $\left|\phi^{\prime}(u)\right| \leq 1$ and $\frac{k(u, v)}{K(u, v)} \geq 1$ or $\frac{k(u, v)}{K(u, v)} \geq \phi^{\prime}(u) \phi^{\prime}(v)$ for $u, v \in[0,1]$, where

$$
k(u, v)=\frac{\partial^{2}}{\partial u \partial v} K(u, v) .
$$

Definitions:

$$
\begin{aligned}
& Q_{0}=\left\{\gamma \neq 0 ; \frac{k(u, v)}{K(u, v)} \geq \frac{\phi^{\prime}(u) \phi^{\prime}(v)}{\gamma^{2}[1-\phi(u) \phi(v)]},(u, v) \in I^{2}\right\}, \\
& Q_{1}=\left\{\gamma \neq 0 ; \phi(u)+A(u, v, \gamma) \geq 0, \phi(v)+B(u, v, \gamma) \geq 0,(u, v) \in I^{2}\right\}, \\
& Q_{2}=\left\{\gamma \neq 0 ;\left|\frac{\phi^{\prime}(u)}{\sqrt{|\gamma|} \frac{\partial}{\partial u} \ln K(u, v)}\right| \leq 1,\left|\frac{\phi^{\prime}(v)}{\sqrt{|\gamma|} \frac{\partial}{\partial v} \ln K(u, v)}\right| \leq 1,(u, v) \in I^{2}\right\},
\end{aligned}
$$

where

$$
A(u, v, \gamma)=\frac{\phi^{\prime}(u)}{\gamma \frac{\partial}{\partial u} \ln K(u, v)}, \quad B(u, v, \gamma)=\frac{\phi^{\prime}(v)}{\gamma \frac{\partial}{\partial v} \ln K(u, v)} .
$$

Theorem 2.1. Let $\phi:[0,1] \rightarrow[-1,1]$ be a monotone and differentiable function with $\phi(1)=0$ and $K(u, v)$ be differentiable with respect to its both arguments $u$ and $v$. Then, $C(u, v ; \theta, \gamma)=K(u, v)[1+\theta \phi(u) \phi(v)]^{\frac{1}{\gamma}}$ is a copula

(i) under Assumptions 1 and 2 for $\theta \in[-1,1]$ provided that $\theta \gamma>0$;

(ii) under Assumption 3 for $\gamma \in(0,1] \cap Q_{0}$ or $\gamma \in(0,1] \cap Q_{1}$ and $-1 \leq \theta \leq 0$;

(iii) under Assumption 1 and 3 and $K(u, v)=K(v, u)$ for all $u, v \in I^{2}$, for $\gamma \in(-\infty,-1] \cap Q_{2}, 0 \leq \theta \leq 1$.

Proof. Obviously boundary conditions are satisfied. Thus, it is sufficient to prove that under the mentioned conditions $C(u, v ; \theta, \gamma)$ is 2-increasing. Let $c(u, v ; \theta, \gamma)=\frac{\partial^{2}}{\partial u \partial v} C(u, v ; \theta, \gamma)$. Hence, we have

$$
\begin{aligned}
& \frac{c(u, v ; \theta, \gamma)}{C(u, v ; \theta, \gamma)} \\
& \quad=\frac{\partial^{2}}{\partial u \partial v} \ln C(u, v ; \theta, \gamma)+\frac{\partial}{\partial u} \ln C(u, v ; \theta, \gamma) \frac{\partial}{\partial v} \ln C(u, v ; \theta, \gamma) .
\end{aligned}
$$

(i) Clearly, since $K(u, v)$ is differentiable, under Assumption 2 we obtain

$$
\frac{\partial}{\partial u} \ln C(u, v ; \theta, \gamma)=\frac{\frac{\partial}{\partial u} \ln K(u, v)}{1+\theta \phi(u) \phi(v)}\left\{1+\theta \phi(v)\left[\phi(u)+\frac{\phi^{\prime}(u)}{\gamma \frac{\partial}{\partial u} \ln K(u, v)}\right]\right\}
$$

and

$$
\frac{\partial}{\partial v} \ln C(u, v ; \theta, \gamma)=\frac{\frac{\partial}{\partial v} \ln K(u, v)}{1+\theta \phi(u) \phi(v)}\left\{1+\theta \phi(v)\left[\phi(u)+\frac{\phi^{\prime}(v)}{\gamma \frac{\partial}{\partial v} \ln K(u, v)}\right]\right\}
$$


are nonnegative. Moreover, by Assumption 1,

$$
\frac{\partial^{2}}{\partial u \partial v} \ln C(u, v ; \theta, \gamma)=\frac{\partial^{2}}{\partial u \partial v} \ln K(u, v)+\frac{\frac{\theta}{\gamma} \phi^{\prime}(u) \phi^{\prime}(v)}{[1+\theta \phi(u) \phi(v)]^{2}}
$$

is nonnegative. Therefore, by (2.3), $c(u, v) \geq 0$ and, thus, by (1.5) the 2 -increasing property holds.

(ii) We prove the case when $\phi(u)$ is nonnegative and decreasing, the other case follows by replacing $\phi(u)$ with $-\phi(u)$ in the copula function. According to (2.3)

$$
\begin{aligned}
\frac{c(u, v ; \theta, \gamma)}{C(u, v ; \theta, \gamma)}= & \frac{\partial^{2}}{\partial u \partial v} \ln K(u, v)+\frac{\frac{\theta}{\gamma} \phi^{\prime}(u) \phi^{\prime}(v)}{[1+\theta \phi(u) \phi(v)]^{2}} \\
& +\left[\frac{\partial}{\partial u} \ln K(u, v)+\frac{\frac{\theta}{\gamma} \phi^{\prime}(u) \phi(v)}{1+\theta \phi(u) \phi(v)}\right] \\
& \times\left[\frac{\partial}{\partial v} \ln K(u, v)+\frac{\frac{\theta}{\gamma} \phi(u) \phi^{\prime}(v)}{1+\theta \phi(u) \phi(v)}\right] \\
= & \frac{k(u, v)}{K(u, v)}+\frac{\theta}{\gamma} \frac{\phi(u) \phi^{\prime}(v)}{1+\theta \phi(u) \phi(v)} \frac{\partial}{\partial u} \ln K(u, v)+\frac{\partial}{\partial v} \ln K(u, v) \\
& \times \frac{\theta \phi^{\prime}(u)}{\gamma[1+\theta \phi(u) \phi(v)]}\left\{\phi(v)+\frac{\phi^{\prime}(v)\left[1+\frac{\theta}{\gamma} \phi(u) \phi(v)\right]}{[1+\theta \phi(u) \phi(v)] \frac{\partial}{\partial v} \ln K(u, v)}\right\},
\end{aligned}
$$

where $k(u, v)=\frac{\partial^{2}}{\partial u \partial v} K(u, v)$. If $0<\gamma \leq 1$, then

$$
\frac{1+\frac{\theta}{\gamma} \phi(u) \phi(v)}{1+\theta \phi(u) \phi(v)}=\frac{1}{\gamma} \frac{\gamma+\theta \phi(u) \phi(v)}{1+\theta \phi(u) \phi(v)} \leq \frac{1}{\gamma}
$$

and since $\phi^{\prime}(u) \leq 0$ and $\theta \leq 0$, we have

$$
\theta \phi^{\prime}(u)\left\{\phi(v)+\frac{\phi^{\prime}(v)\left[1+\frac{\theta}{\gamma} \phi(u) \phi(v)\right]}{[1+\theta \phi(u) \phi(v)] \frac{\partial}{\partial v} \ln K(u, v)}\right\} \geq \theta \phi^{\prime}(u)\{\phi(v)+B(u, v, \gamma)\} \text {. }
$$

Consequently, if $\gamma \in(0,1] \cap Q_{1}$

$$
\begin{aligned}
\frac{c(u, v ; \theta, \gamma)}{C(u, v ; \theta, \gamma)} \geq & \frac{k(u, v)}{K(u, v)}+\frac{\theta}{\gamma} \frac{\phi(u) \phi^{\prime}(v)}{1+\theta \phi(u) \phi(v)} \frac{\partial}{\partial u} \ln K(u, v) \\
& +\frac{\partial}{\partial v} \ln K(u, v) \frac{\theta \phi^{\prime}(u)}{\gamma[1+\theta \phi(u) \phi(v)]}\{\phi(v)+B(u, v, \gamma)\} \geq 0,
\end{aligned}
$$

and if $\gamma \in(0,1] \cap Q_{0}$ we can rewrite

$$
\begin{aligned}
\frac{c(u, v ; \theta, \gamma)}{C(u, v ; \theta, \gamma)} \geq & \frac{k(u, v)}{K(u, v)}+\frac{\theta}{\gamma} \frac{\phi(u) \phi^{\prime}(v)}{1+\theta \phi(u) \phi(v)} \frac{\partial}{\partial u} \ln K(u, v) \\
& +\frac{\theta}{\gamma} \frac{\phi^{\prime}(u) \phi(v)}{1+\theta \phi(u) \phi(v)} \frac{\partial}{\partial v} \ln K(u, v)+\frac{\theta \phi^{\prime}(u) \phi^{\prime}(v)}{\gamma^{2}[1+\theta \phi(u) \phi(v)]} \geq 0
\end{aligned}
$$


because

$$
\frac{\theta \phi^{\prime}(u) \phi^{\prime}(v)}{\gamma^{2}[1+\theta \phi(u) \phi(v)]} \geq \frac{-\phi^{\prime}(u) \phi^{\prime}(v)}{\gamma^{2}[1-\phi(u) \phi(v)]} .
$$

(iii) Similar to part (b), we just prove the case when $\phi(u)$ is nonnegative and decreasing. Since $\gamma \in(-\infty,-1) \cap Q_{2}$, it follows that $\phi(u)+\frac{\phi^{\prime}(u)}{\gamma \frac{\partial}{\partial u} \ln K(u, v)} \geq 0$. Thus, we have

$$
\begin{aligned}
\frac{c(u, v ; \theta, \gamma)}{C(u, v ; \theta, \gamma)}= & \frac{\partial^{2}}{\partial u \partial v} \ln K(u, v)+\frac{\frac{\theta}{\gamma} \phi^{\prime}(u) \phi^{\prime}(v)}{[1+\theta \phi(u) \phi(v)]^{2}} \\
& +\{1+\theta \phi(v)[\phi(u)+A(u, v, \gamma)]\} \\
& \times\{1+\theta \phi(u)[\phi(v)+B(u, v, \gamma)]\} \\
& \times \frac{\frac{\partial}{\partial u} \ln K(u, v) \frac{\partial}{\partial v} \ln K(u, v)}{[1+\theta \phi(u) \phi(v)]^{2}} \\
\geq & \frac{\partial^{2}}{\partial u \partial v} \ln K(u, v)+\frac{\frac{\theta}{\gamma} \phi^{\prime}(u) \phi^{\prime}(v)}{[1+\theta \phi(u) \phi(v)]^{2}} \\
& +\frac{\frac{\partial}{\partial u} \ln K(u, v) \frac{\partial}{\partial v} \ln K(u, v)}{[1+\theta \phi(u) \phi(v)]^{2}} \\
= & \frac{\partial^{2}}{\partial u \partial v} \ln K(u, v)+\frac{\frac{\partial}{\partial u} \ln K(u, v) \frac{\partial}{\partial v} \ln K(u, v)}{[1+\phi(u) \phi(v)]^{2}} \\
& \times\left\{1-\theta \frac{\phi^{\prime}(u)}{\sqrt{|\gamma|} \frac{\partial}{\partial u} \ln K(u, v)} \frac{\phi^{\prime}(v)}{\sqrt{|\gamma|} \frac{\partial}{\partial v} \ln K(u, v)}\right\} \geq 0
\end{aligned}
$$

This completes the proof.

Theorem 2.2. Let $\phi$ be a differentiable function and $\phi(1)=0$. Then, $C(u, v ; \theta)=$ $K(u, v) e^{\theta \phi(u) \phi(v)}$ is a copula

(i) for $\theta \in[0,1]$ under Assumptions 1 and 4;

(ii) for $\theta \in[-1,0]$ under Assumptions 3 and 5 .

Proof. (i) Obviously boundary conditions are satisfied. As before, it is sufficient to show that $c(u, v) \geq 0$. According to Assumption 4, we have

$$
\frac{\partial^{2}}{\partial u \partial v} \ln C(u, v ; \theta)=\frac{\partial^{2}}{\partial u \partial v} \ln K(u, v)+\theta \phi^{\prime}(u) \phi^{\prime}(v) \geq 0
$$

and since $\theta \phi(v) \leq 0$ we obtain

$$
\theta \phi^{\prime}(u) \phi(v) \geq \theta \phi(v) \frac{\partial}{\partial u} \ln K(u, v) .
$$


Thus,

$$
\frac{\partial}{\partial u} \ln C(u, v ; \theta) \geq \frac{\partial}{\partial u} \ln K(u, v)[1+\theta \phi(v)] \geq 0,
$$

and thus, (2.3) is nonnegative. Hence, the result follows.

(ii) Again, we have

$$
\begin{aligned}
\frac{c(u, v ; \theta)}{C(u, v ; \theta)}= & \frac{k(u, v)}{K(u, v)}-\frac{\partial}{\partial u} \ln K(u, v) \frac{\partial}{\partial v} \ln K(u, v)+\theta \phi^{\prime}(u) \phi^{\prime}(v) \\
& +\frac{\partial}{\partial u} \ln K(u, v) \frac{\partial}{\partial v} \ln K(u, v)\left[1+\theta \frac{\phi^{\prime}(u) \phi(v)}{\frac{\partial}{\partial u} \ln K(u, v)}\right] \\
& \times\left[1+\theta \frac{\phi(u) \phi^{\prime}(v)}{\frac{\partial}{\partial v} \ln K(u, v)}\right] .
\end{aligned}
$$

Since $\theta \in[-1,0]$, by the properties of $\phi(u)$, we obtain

$$
\frac{c(u, v ; \theta)}{C(u, v ; \theta)} \geq \frac{k(u, v)}{K(u, v)}+\theta \phi^{\prime}(u) \phi^{\prime}(v) \geq 0
$$

that is, $c(u, v) \geq 0$.

\section{Some examples}

In this section, we introduce several members of our class of copulas. First, we need to recall some copulas $K(u, v)$ in Table 1 which are used in the sequel.

Table 1 Some bivariate copulas recalled

\begin{tabular}{lc}
\hline$K(u, v)$ & Parameter spaces \\
\hline$u v\left[1+\delta(1-u)^{p}(1-v)^{p}\right]$ & $p>0,-1 \leq \delta \leq\left(\frac{p+1}{p-1}\right)^{p-1}$ \\
$u v\left[1+\delta\left(u^{n}-1\right)\left(v^{n}-1\right)\right]$ & $($ see Huang and Kotz $(1999))$ \\
& $n \in \aleph,-\frac{1}{n^{2}} \leq \delta \leq 1$ \\
$u v\left[1+\delta\left(1-u^{p}\right)\left(1-v^{p}\right)\right]$ & $(\operatorname{see~Grane~}(2009))$ \\
& $p>0,-(\max \{1, p\})^{-2} \leq \delta \leq \frac{1}{p}$ \\
$u v\left[1+\alpha(1-u)(1-v)+\beta(1-u)^{2}(1-v)^{2}\right]$ & $(\operatorname{see~Huang~and~Kotz~}(1999))$ \\
& $|\alpha| \leq 1, \alpha+\beta \geq-1, \beta \leq \frac{3-\alpha+\sqrt{9-6 \alpha-3 \alpha^{2}}}{2}$ \\
$u v[1+\alpha(1-u)(1-v)+\beta u v(1-u)(1-v)]$ & $(\operatorname{see~Lin~}(1987))$ \\
& $|\alpha| \leq 1, \alpha+\beta \geq-1, \beta \leq \frac{3-\alpha+\sqrt{9-6 \alpha-3 \alpha^{2}}}{2}$ \\
& $(\operatorname{see~Lin~}(1987))$ \\
\hline
\end{tabular}


Example 3.1. Based on Theorem 2.1(i) the bivariate function

$$
\begin{aligned}
& C(u, v ; \theta, \gamma, \delta, k) \\
& \quad=u v\left[1+\delta(1-u)^{2}(1-v)^{2}\right]\left[1+\theta\left(1-u^{k}\right)\left(1-v^{k}\right)\right]^{\frac{1}{\gamma}},
\end{aligned}
$$

is a copula for $u, v \in I$ when

$$
\text { (a) } 0 \leq \theta \leq 1, \quad 0 \leq \delta \leq 1, \quad 0 \leq k \leq 1, \quad \gamma \geq 1
$$

or

$$
\text { (b) } 0 \leq \theta \leq 1, \quad 0 \leq \delta \leq 2, \quad 1 \leq k \leq 3, \quad \gamma \geq 3
$$

and for $-1 \leq \theta \leq 0,0 \leq \delta \leq 1$ when

$$
\text { (c) } 0 \leq k \leq 1, \quad \gamma \leq-2
$$

or

$$
\text { (d) } 1 \leq k \leq 2, \quad \gamma \leq-4 \text {, }
$$

or

$$
\text { (e) } 2 \leq k \leq 3, \quad \gamma \leq-6 \text {. }
$$

Here, $\phi(u)=1-u^{k}$, for which boundary condition of Theorem 2.1 holds. Moreover, by using Table 1

$$
K(u, v ; \delta)=u v\left[1+\delta(1-u)^{2}(1-v)^{2}\right], \quad \delta \in[-1,3],
$$

is a copula and

$$
\frac{\partial^{2}}{\partial v \partial u} \ln K(u, v ; \delta)=\frac{4 \delta(1-u)(1-v)}{\left[1+\delta(1-u)^{2}(1-v)^{2}\right]^{2}} \geq 0, \quad \delta \in[0,3]
$$

for $\forall u, v \in I$. Now, we define

$$
\begin{aligned}
h(u, v ; \delta, \gamma, k) & =\phi(u)+\frac{\phi^{\prime}(u)}{\gamma \frac{\partial}{\partial u} \ln K(u, v ; \delta)} \\
& =1-u^{k}-\frac{k u^{k}\left[1+\delta(1-u)^{2}(1-v)^{2}\right]}{\gamma\left[1+\delta(1-u)^{2}(1-v)^{2}-2 \delta u(1-u)(1-v)^{2}\right]} \\
& =1-u^{k} f(u, v ; \delta, \gamma, k),
\end{aligned}
$$

where

$$
f(u, v ; \delta, \gamma, k)=1+\frac{k\left(1+\delta(1-u)^{2}(1-v)^{2}\right)}{\gamma\left[1+\delta(1-u)^{2}(1-v)^{2}-2 \delta u(1-u)(1-v)^{2}\right]} .
$$

(a) In this case, since $f(u, v ; \delta, \gamma, k) \geq 1$, it is sufficient to show that

$$
u^{k} f(u, v ; \delta, \gamma, k) \leq 2 \text {. }
$$




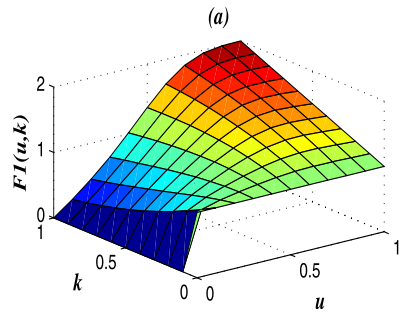

(d)

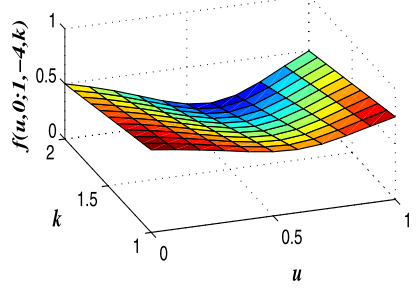

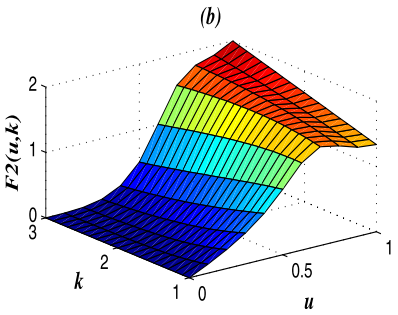

(e)

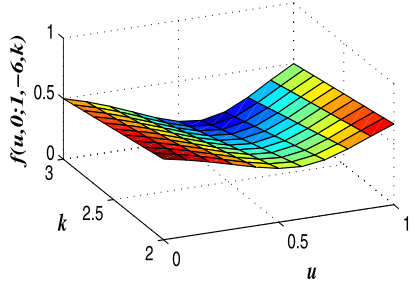

(c)

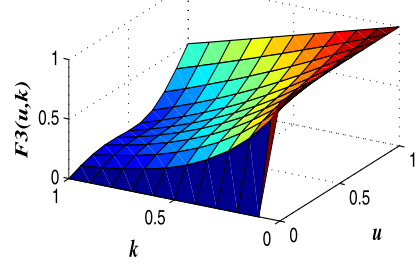

$(f)$

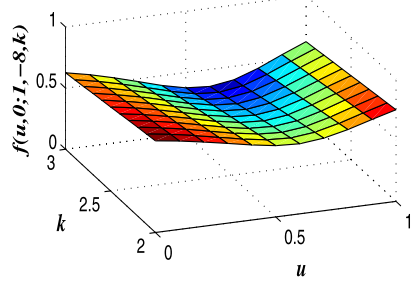

Figure 1 Illustrations of $F_{i}(u, k)$ for $i=1,2,3$ and $f(u, 0 ; 1, \gamma, k)$ for $\gamma=-4,-6,-8$.

On the other hand, $f(u, v ; \delta, \gamma, k)$ is decreasing in $\gamma$ and $v$, so $f(u, v ; \delta, \gamma, k) \leq$ $f(u, 0 ; \delta, 1, k)$ for $u \in[0,1], \delta \in[0,1]$ and $k \in[0,1]$ and $f(u, 0 ; \delta, 1, k)$ is increasing function in $\delta$, because

$$
\frac{\partial}{\partial \delta} f(u, 0 ; \delta, 1, k)=\frac{2 k(1-u) u}{\left(1+\delta-4 \delta u+3 \delta u^{2}\right)^{2}} \geq 0 .
$$

So, the maximum of $f(u, v ; \delta, \gamma, k)$ happens at $v=0, \gamma=1$ and $\delta=1$. Moreover, As we see in Figure $1(\mathrm{a}), 0 \leq F_{1}(u, k)=u^{k} f(u, 0 ; 1,1, k) \leq 2$ for $u \in[0,1], k \in$ $[0,1]$. Thus, we can conclude that $0 \leq u^{k} f(u, v ; \delta, \gamma, k) \leq 2$ for $u, v \in[0,1], 0 \leq$ $\delta \leq 1,0 \leq k \leq 1$ and $\gamma \geq 1$.

(b) By a similar argument, we can show that $0 \leq F_{2}(u, k)=u^{k} f(u, 0 ; 2,3, k) \leq$ 2. Figure 1(b) confirms this claim for $0 \leq u \leq 1$ and $1 \leq k \leq 3$.

(c) In this case, since $f(u, v ; \delta, \gamma, k) \leq 1$, it is sufficient to show that $f(u, v$; $\delta, \gamma, k) \geq 0$. By a similar argument, we just investigate $f(u, v ; \delta, \gamma, k)$ at $v=0$, $\gamma=-2$ and $\delta=1$. Figure 1(c) illustrates $F_{3}(u, k)=f(u, 0 ; 1,-2, k) \geq 0$ for $0 \leq$ $u \leq 1$ and $0 \leq k \leq 1$.

Other cases are similarly proved. Figure 1(d)-(f) illustrates that $0 \leq f(u, 0$; $1, \gamma, k) \leq 1$ for certain values of $\gamma$.

Example 3.2. Based on Theorem 2.1(i) the bivariate function

$$
\begin{aligned}
& C(u, v ; \theta, \gamma, \alpha, k) \\
& \quad=u v\left[1+\alpha\left(u^{2}-1\right)\left(v^{2}-1\right)\right]\left[1+\theta\left(1-u^{k}\right)\left(1-v^{k}\right)\right]^{1 / \gamma}
\end{aligned}
$$


is a copula for

$$
-1 \leq \theta \leq 0, \quad 0 \leq \alpha \leq 0.4, \quad \gamma \leq-5 k, \quad k>0,
$$

or

$$
0 \leq \theta \leq 1, \quad 0 \leq \alpha \leq 0.4, \quad \gamma \geq 5 k, \quad k>0 .
$$

First, we note that

$$
K(u, v ; \alpha)=u v\left[1+\alpha\left(u^{2}-1\right)\left(v^{2}-1\right)\right],
$$

is a copula for $\alpha \in[-0.25,1]$ and

$$
\frac{\partial^{2}}{\partial v \partial u} \ln K(u, v ; \alpha)=\frac{4 \alpha u v}{\left[1+\alpha\left(u^{2}-1\right)\left(v^{2}-1\right)\right]^{2}} \geq 0, \quad \alpha \in[0,1] .
$$

Now, we define

$$
r(u, v ; \alpha, \gamma, k)=\phi(u)+\frac{\phi^{\prime}(u)}{\gamma \frac{\partial}{\partial u} \ln K(u, v ; \alpha)}=1-u^{k}\left\{1+\frac{k}{\gamma} R(u, v ; \alpha)\right\},
$$

where

$$
R(u, v ; \alpha)=\frac{1+\alpha\left(u^{2}-1\right)\left(v^{2}-1\right)}{1+\alpha\left(u^{2}-1\right)\left(v^{2}-1\right)+2 \alpha u^{2}\left(v^{2}-1\right)} .
$$

We note that it is sufficient to investigate the range of $r(u, v ; \alpha, \gamma, k)$ at $\gamma=-5 k$ and $\gamma=5 k$. Moreover, we can easily show that the function $R(u, v ; \alpha)$ is a decreasing function in $v$ and increasing function in $\alpha$ and $u$, so $1 \leq R(u, v ; \alpha) \leq 5$. Hence,

$$
\begin{aligned}
0 \leq u^{k}\left\{1-\frac{1}{5} R(u, v ; \alpha)\right\} \leq 1, \\
u, v \in[0,1] \Rightarrow 0 \leq r(u, v ; \alpha, \gamma, k) \leq 1, \\
0 \leq u^{k}\left\{1+\frac{1}{5} R(u, v ; \alpha)\right\} \leq 2, \\
u, v \in[0,1] \quad \Rightarrow \quad-1 \leq r(u, v ; \alpha, \gamma, k) \leq 1 .
\end{aligned}
$$

for $\alpha \in[0,0.4]$. Figure 2 illustrates our claim for certain values of the parameters.

It is also worth noting that, similarly, the bivariate function

$$
\begin{aligned}
& C(u, v ; \delta, \theta, \gamma, p) \\
& \quad=u v\left[1+\delta\left(1-u^{p}\right)\left(1-v^{p}\right)\right][1+\theta(1-u)(1-v)]^{\frac{1}{\gamma}},
\end{aligned}
$$



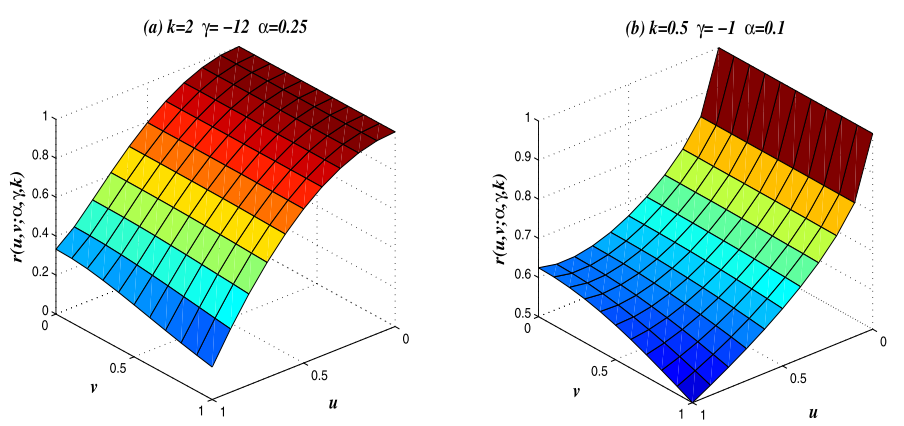

Figure 2 Illustrations of $r(u, v ; \alpha, \gamma, k)$.

is a copula for $p>0,0 \leq \delta \leq \frac{1}{2 p}, \gamma \geq 2$ and $0 \leq \theta \leq 1$ or $\gamma \leq-2$ and $-1 \leq \theta \leq 0$. Also, the bivariate function

$$
\begin{aligned}
C(u, v ; \alpha, \beta, \theta, \gamma)= & u v\left[1+\alpha(1-u)(1-v)+\beta(1-u)^{2}(1-v)^{2}\right] \\
& \times\left[1+\theta\left(e^{-u}-e^{-1}\right)\left(e^{-v}-e^{-1}\right)\right]^{\frac{1}{\gamma}},
\end{aligned}
$$

is also a copula for $0 \leq \beta \leq 1,0 \leq \alpha<1,-1 \leq \theta \leq 0$ and $\gamma \leq-\frac{2}{e(1-\alpha)}$ or $0 \leq$ $\theta \leq 1$ and $\gamma \geq \frac{2}{e(1-\alpha)}$ and the bivariate function

$$
\begin{aligned}
C(u, v ; \alpha, \beta, \theta, \gamma, k)= & u v\left[1+\alpha(1-u)(1-v)+\beta(1-u)^{2}(1-v)^{2}\right] \\
& \times[1+\theta(1-u)(1-v)]^{\frac{1}{\gamma}},
\end{aligned}
$$

is a copula for $0 \leq \alpha<1,0 \leq \beta \leq 1,-1 \leq \theta \leq 0, \gamma \leq-\frac{2}{1-\alpha}$ or $0 \leq \theta \leq 1$, $\gamma \geq \frac{2}{1-\alpha}$.

Example 3.3. The bivariate function

$$
C(u, v ; \theta, \delta, \gamma)=u v\left[1+\delta(1-u)^{2}(1-v)^{2}\right][1+\theta(1-u)(1-v)]^{\frac{1}{\gamma}},
$$

is a copula for $u, v \in I, 0 \leq \theta \leq 1,0 \leq \delta \leq 1.5$ and $\gamma \leq-3$ because it satisfies the conditions of Theorem 2.1(ii).

Clearly, since $\phi(u)=1-u$, boundary condition and Assumption 3 hold. Furthermore, similar to Example 3.1, we have

Now, we define

$$
\frac{\partial^{2}}{\partial v \partial u} \ln K(u, v ; \theta, \delta, \gamma) \geq 0, \quad \delta \in[0,1.5]
$$

$$
\begin{aligned}
t(u, v ; \delta, \gamma) & =\frac{-\phi^{\prime}(u)}{\sqrt{|\gamma|} \frac{\partial}{\partial u} \ln K(u, v)} \\
& =\frac{u\left[1+\delta(1-u)^{2}(1-v)^{2}\right]}{\sqrt{|\gamma|}\left[1+\delta(1-u)^{2}(1-v)^{2}-2 \delta u(1-u)(1-v)^{2}\right]},
\end{aligned}
$$




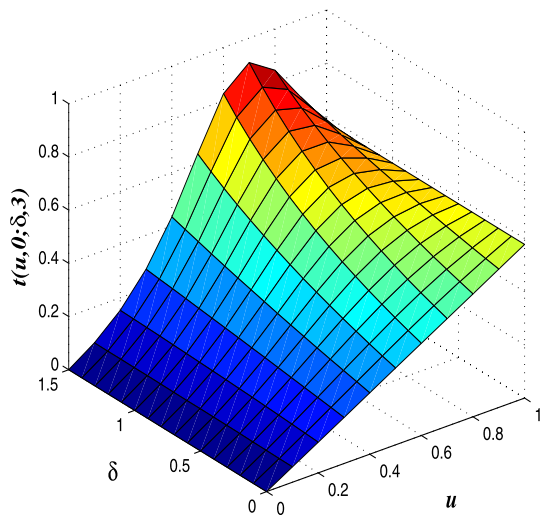

Figure 3 Illustrations of $t(u, 0 ; \delta,-3)$.

which is obviously nonnegative. Thus, it is sufficient to show that $t(u, v ; \delta, \gamma) \leq$ 1. Obviously $t(u, v ; \delta, \gamma)$ is a decreasing function in $v$ and $\gamma$. So, we need to investigate the values of $t(u, 0 ; \delta,-3)$. As Figure 3 illustrates we can show that $t(u, 0 ; \delta,-3) \leq 1$ for $0 \leq u \leq 1$ and $0 \leq \delta \leq 1.5$.

Example 3.4. The bivariate function

$$
C(u, v ; \theta, \alpha)=u v[1+\alpha(1-u)(1-v)(1+u v)] e^{\theta\left(e^{-1}-e^{-u}\right)\left(e^{-1}-e^{-v}\right)},
$$

is a copula for $0 \leq \theta \leq 1$ and $0 \leq \alpha \leq 1-e^{-1}$, because it satisfies the conditions of Theorem 2.2(i). Clearly, since $\phi(u)=e^{-1}-e^{-u}$, the boundary condition holds. Furthermore, by using Table 1, we have that

$$
K(u, v ; \alpha)=u v[1+\alpha(1-u)(1-v)(1+u v)],
$$

is a copula for $-0.5 \leq \alpha \leq 1$ and

$$
\frac{\partial^{2}}{\partial v \partial u} \ln K(u, v ; \alpha)=\frac{\alpha\left[1+\alpha(1-u)^{2}(1-v)^{2}+(1-2 u)(1-2 v)\right]}{[1+\alpha(1-u)(1-v)(1+u v)]^{2}} \geq 0,
$$

for $\alpha \in[0,1]$, because $(1-2 u)(1-2 v) \geq-1$. Now, we define

$$
\begin{aligned}
q(u, v ; \alpha) & =\frac{\partial}{\partial u} \ln K(u, v ; \alpha)-\phi^{\prime}(u) \\
& =\frac{1}{u}+\frac{\alpha(1-v)(v-2 u v-1)}{1+\alpha(1-u)(1-v)(1+u v)}-e^{-u} \\
& =g(u)+f_{1}(u, v ; \alpha),
\end{aligned}
$$

where, obviously

$$
g(u)=\frac{1}{u}-e^{-u} \geq 0, \quad f_{1}(u, v ; \alpha)=\frac{\alpha(1-v)(v-2 u v-1)}{1+\alpha(1-u)(1-v)(1+u v)} \leq 0 .
$$


$\alpha=0.3$

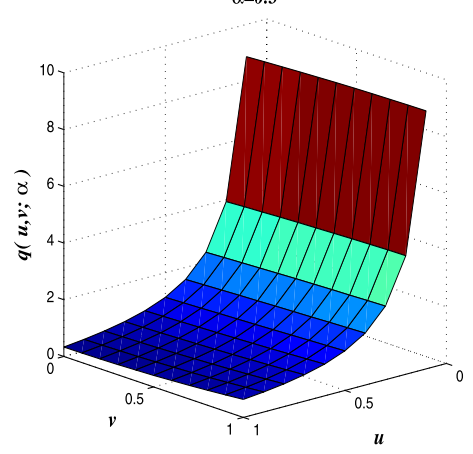

$\alpha=0.6$

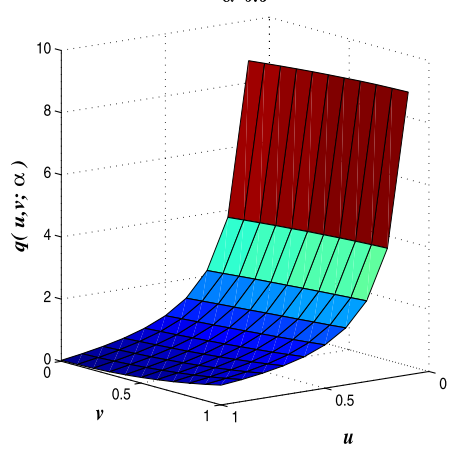

Figure 4 Illustration of $q(u, v, \alpha)$.

Both functions $g$ and $f_{1}$ are decreasing in $u$, because

$$
\begin{aligned}
g^{\prime}(u)= & e^{-u}-\frac{1}{u^{2}} \leq e^{-u}-1 \leq 0 \\
\frac{\partial}{\partial u} f_{1}(u, v ; \alpha)= & \frac{1}{[1+\alpha(1-u)(1-v)(1+u v)]^{2}}\left[-\alpha^{2}(1-v)^{2}(v-2 u v-1)^{2}\right. \\
& -2 \alpha v(1-v)(1+\alpha(1-u)(1-v)(1+u v))] \leq 0 .
\end{aligned}
$$

$g$ decreases very faster than $f_{1}$, so to find values $\alpha$ such that $q(u, v ; \alpha) \geq 0$ for $u, v \in[0,1]$, it is sufficient to investigate the sign of $q(1, v ; \alpha)$ for $v \in[0,1]$. But, clearly, we have

$$
q(1, v ; \alpha)=1-e^{-1}-\alpha\left(1-v^{2}\right) \geq 0, \quad 0 \leq \alpha \leq 1-e^{-1}
$$

Figure 4 also confirms the validity of Assumption 4 for $\alpha=0.3$ and 0.6.

Example 3.5. By Theorem 2.2(i), the bivariate function

$$
\begin{aligned}
C(u, v ; \alpha, \beta, \theta)= & u v\left[1+\alpha(1-u)(1-v)+\beta(1-u)^{2}(1-v)^{2}\right] \\
& \times e^{\theta\left(e^{-1}-e^{-u}\right)\left(e^{-1}-e^{-v}\right)}
\end{aligned}
$$

is a copula for $0 \leq \theta \leq 1,0 \leq \beta \leq 0.5$ and $0 \leq \alpha \leq 1-e^{-1}$. Similar to Example $3.3, \phi(u)=e^{-1}-e^{-u}$ and the boundary condition holds and

$$
K(u, v ; \alpha, \beta)=u v\left[1+\alpha(1-u)(1-v)+\beta(1-u)^{2}(1-v)^{2}\right]
$$

is a copula for $|\alpha| \leq 1, \alpha+\beta \geq-1$ and $\beta \leq \frac{3-\alpha+\sqrt{9-6 \alpha-3 \alpha^{2}}}{2}$ with

$$
\frac{\partial^{2}}{\partial v \partial u} \ln K(u, v ; \alpha, \beta)=\frac{\alpha+4 \beta(1-u)(1-v)+\alpha \beta(1-u)^{2}(1-v)^{2}}{\left[1+\alpha(1-u)(1-v)+\beta(1-u)^{2}(1-v)^{2}\right]^{2}} \geq 0,
$$


for $0 \leq \alpha \leq 1$ and $0 \leq \beta \leq \frac{3-\alpha+\sqrt{9-6 \alpha-3 \alpha^{2}}}{2}$. Now, we define

$$
\begin{aligned}
s(u, v ; \alpha, \beta) & =\frac{\partial}{\partial u} \ln K(u, v ; \alpha, \beta)-e^{-u} \\
& =\frac{1}{u}-\frac{\alpha(1-v)+2 \beta(1-u)(1-v)^{2}}{1+\alpha(1-u)(1-v)+\beta(1-u)^{2}(1-v)^{2}}-e^{-u} \\
& =g(u)-f_{2}(u, v ; \alpha, \beta),
\end{aligned}
$$

where $g(u)=\frac{1}{u}-e^{-u}>0$ and

$$
f_{2}(u, v ; \alpha, \beta)=\frac{\alpha(1-v)+2 \beta(1-u)(1-v)^{2}}{1+\alpha(1-u)(1-v)+\beta(1-u)^{2}(1-v)^{2}} \geq 0
$$

for $0 \leq \alpha \leq 1$ and $0 \leq \beta \leq \frac{3-\alpha+\sqrt{9-6 \alpha-3 \alpha^{2}}}{2}$. $f_{2}$ is a decreasing function in $v$ for $0 \leq \alpha \leq 1$ and $0 \leq \beta \leq 1$, because

$$
\begin{aligned}
\frac{\partial}{\partial v} f_{2}(u, v ; \alpha, \beta) & \\
& =-\frac{\alpha+4 \beta(1-u)(1-v)}{A}+\frac{(1-u)(1-v)(\alpha+2 \beta(1-u)(1-v))}{A^{2}} \\
& \leq-\frac{\alpha+2 \beta(1-u)(1-v)}{A}+\frac{(1-u)(1-v)(\alpha+2 \beta(1-u)(1-v))}{A^{2}} \\
& =\frac{\alpha+2 \beta(1-u)(1-v)}{A}\left[-1+\frac{\beta(1-u)^{2}(1-v)^{2}}{A}\right] \leq 0,
\end{aligned}
$$

where $A=1+\alpha(1-u)(1-v)+\beta(1-u)^{2}(1-v)^{2}>0$. So, it is sufficient to investigate the behavior of $f_{2}(u, 0 ; \alpha, \beta)$. This function will be increasing, decreasing or upsidedown U-shaped in $u$, depending on different values of $\alpha$ and $\beta$. Thus, we consider two cases: (i) $f_{2}(u, 0 ; \alpha, \beta)$ is an increasing or decreasing function and (ii) $f_{2}(u, 0 ; \alpha, \beta)$ is upsidedown U-shaped function in $u$.

For case (i), since $g$ is decreasing very fast, we just investigate the sign of $s(u, 0 ; \alpha, \beta)$ for $u=0$ and $u=1$ and conclude that $s(u, 0 ; \alpha, \beta) \geq 0$ for $u, v \in$ $[0,1], 0 \leq \alpha \leq 1-e^{-1}$.

In case (ii), we note that $f_{2}$ is an increasing function in $\alpha$ and $\beta$. So, we define

$$
h(u)=f_{2}\left(u, 0 ; 1-e^{-1}, 0.5\right)=\frac{2-u-e^{-1}}{1+\left(1-e^{-1}\right)(1-u)+0.5(1-u)^{2}} .
$$

$h(u)$ has a maximum at $u_{0}=0.3670$ and

$$
h(u) \geq \frac{\alpha+2 \beta(1-u)}{1+\alpha(1-u)+\beta(1-u)^{2}}, \quad \alpha \in\left[0,1-e^{-1}\right], \beta \in[0,0.5] .
$$



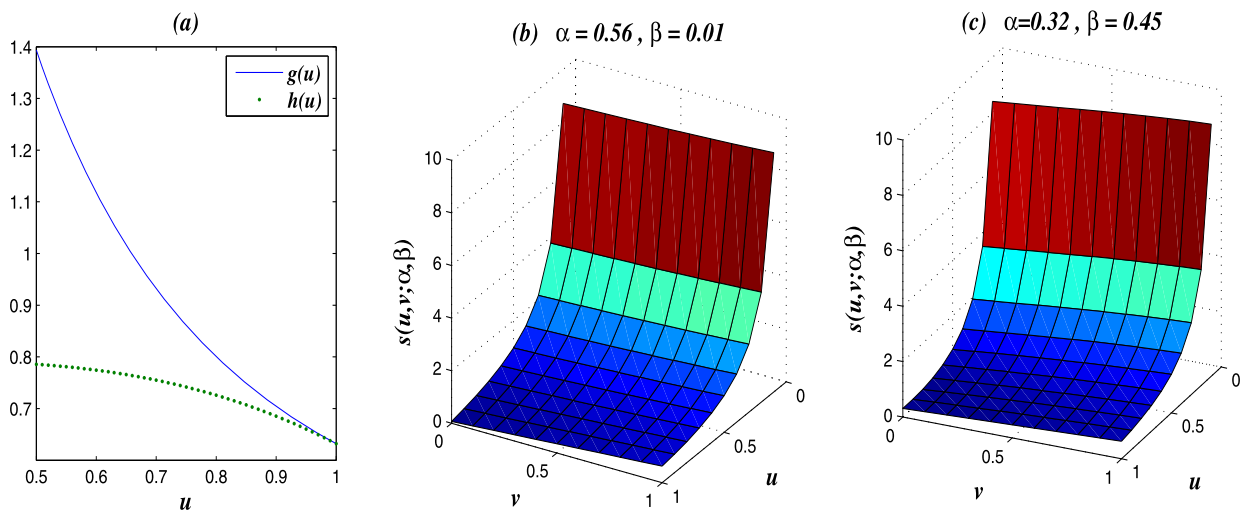

Figure 5 Illustrations of $s(u, v ; \alpha, \beta)$.

Thus, since $g(u)$ is decreasing very fast compared to $h(u)$, we just plot $g(u)$ and $h(u)$ for $u \in[0.5,1]$, in Figure 5(a). Then, we have

$$
g(u)-h(u) \geq 0, \quad \forall u \in[0,1] .
$$

Hence, we conclude that $s(u, v ; \alpha, \beta) \geq 0$ for $\alpha \in\left[0,1-e^{-1}\right]$ and $\beta \in[0,0.5]$. Moreover, Figure 5(b)-(c) confirms the validity of Assumption 4 of Theorem 2.2(a) for certain values of the parameters.

Example 3.6. The bivariate function

$$
\begin{aligned}
C(u, v ; \theta, \alpha, \beta)= & u v\left[1+\alpha(1-u)(1-v)+\beta(1-u)^{2}(1-v)^{2}\right] \\
& \times e^{\theta(1-u)(1-v)},
\end{aligned}
$$

is a copula for $\theta \in[-1,0], \alpha \in[0,1]$ and $\beta \in[-1,1]$. In this example, $\phi(u)=$ $1-u$ and clearly the boundary condition and Assumption 3 of Theorem 2.2(ii) hold and

$$
K(u, v ; \alpha, \beta)=u v\left[1+\alpha(1-u)(1-v)+\beta(1-u)^{2}(1-v)^{2}\right]
$$

is a copula for $|\alpha| \leq 1, \alpha+\beta \geq-1$ and $\beta \leq \frac{3-\alpha+\sqrt{9-6 \alpha-3 \alpha^{2}}}{2}$. Now, to check the validity of Assumption 5, we define

$$
\begin{aligned}
g(u, v ; \alpha, \beta) & =\frac{k(u, v ; \alpha, \beta)}{K(u, v ; \alpha, \beta)}-1 \\
& =\frac{k(u, v ; \alpha, \beta)-K(u, v ; \alpha, \beta)}{K(u, v ; \alpha, \beta)} \\
& =\frac{1}{K(u, v ; \alpha, \beta)}\left\{1+\alpha(1-u)(1-v)+\beta(1-u)^{2}(1-v)^{2}\right.
\end{aligned}
$$




$$
\begin{aligned}
& -(u+v-2 u v)[\alpha+2 \beta(1-u)(1-v)] \\
& +u v[\alpha+4 \beta(1-u)(1-v)] \\
& \left.-u v\left[1+\alpha(1-u)(1-v)+\beta(1-u)^{2}(1-v)^{2}\right]\right\} .
\end{aligned}
$$

First, let $0 \leq \beta \leq 1$, we have

$$
g(u, v ; \alpha, \beta)=\frac{f_{1}(u, v ; \alpha, \beta)-f_{2}(u, v ; \alpha, \beta)}{K(u, v ; \alpha, \beta)}=\frac{F(u, v ; \alpha, \beta)}{K(u, v ; \alpha, \beta)},
$$

where

$$
\begin{aligned}
f_{1}(u, v ; \alpha, \beta)= & 1+\alpha(1-u)(1-v)+\beta(1-u)^{2}(1-v)^{2} \\
& +u v[\alpha+4 \beta(1-u)(1-v)] \geq 0, \\
f_{2}(u, v ; \alpha, \beta)= & u v\left[1+\alpha(1-u)(1-v)+\beta(1-u)^{2}(1-v)^{2}\right] \\
& +(u+v-2 u v)[\alpha+2 \beta(1-u)(1-v)] \geq 0
\end{aligned}
$$

and

$$
F(u, v ; \alpha, \beta)=f_{1}(u, v ; \alpha, \beta)-f_{2}(u, v ; \alpha, \beta) .
$$

$f_{1}$ and $f_{2}$ are increasing functions in $\alpha$ and $\beta$. Moreover, $f_{1}$ and $f_{2}$ are linear functions of $\alpha$ and $\beta$. So, to find the signs of $g$, it is sufficient to investigate the signs of $F(u, v ; 0,0)$ and $F(u, v ; 1,1)$. Hence, we have

$$
\begin{aligned}
F(u, v ; 0,0)= & 1-u v \geq 0, \quad u, v \in[0,1] \\
F(u, v ; 1,1)= & {\left[1+(1-u)(1-v)+(1-u)^{2}(1-v)^{2}\right](1-u v)+4 u v } \\
& \times(1-u)(1-v)-[1+2(1-u)(1-v)](u+v-2 u v)+u v \\
\geq & 0, \quad u, v \in[0,1] .
\end{aligned}
$$

Figure 6(a) illustrates $F(u, v ; 1,1) \geq 0$ for $u, v \in[0,1]$. Thus, $g \geq 0$ and hence Assumption 5 holds for $0 \leq \beta \leq 1$.

Now, we let $-1 \leq \beta \leq 0$ and

$$
g(u, v ; \alpha, \beta)=\frac{f_{3}(u, v ; \alpha, \beta)-f_{4}(u, v ; \alpha, \beta)}{K(u, v ; \alpha, \beta)}=\frac{G(u, v ; \alpha, \beta)}{K(u, v ; \alpha, \beta)},
$$

where

$$
\begin{aligned}
f_{3}(u, v ; \alpha, \beta)= & 1+\alpha(1-u)(1-v)+\alpha u v-2 \beta(1-u)(1-v) \\
& \times(u+v-2 u v)-\beta u v(1-u)^{2}(1-v)^{2} \geq 0 \\
f_{4}(u, v ; \alpha, \beta)= & \alpha(u+v-2 u v)+u v[1+\alpha(1-u)(1-v)] \\
& -\beta(1-u)^{2}(1-v)^{2}-4 \beta u v(1-u)(1-v) \geq 0
\end{aligned}
$$


(a)

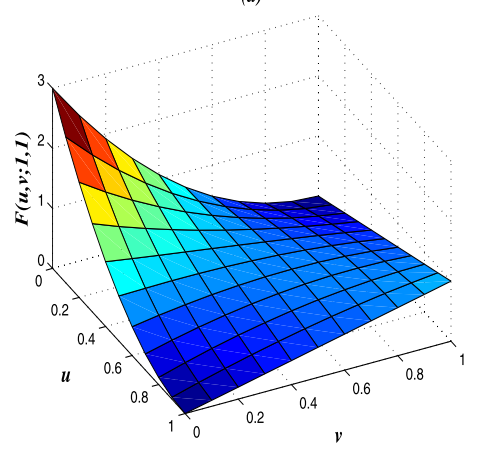

(b)

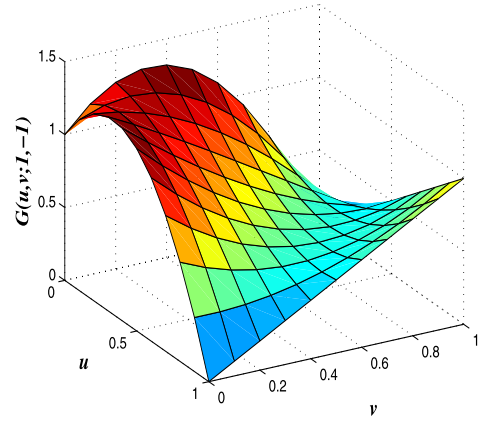

Figure 6 Illustrations of $F(u, v ; 1,1)$ and $G(u, v ; 1,-1)$.

and

$$
G(u, v ; \alpha, \beta)=f_{3}(u, v ; \alpha, \beta)-f_{4}(u, v ; \alpha, \beta) .
$$

$f_{3}$ and $f_{4}$ are increasing functions in $\alpha$ and decreasing functions in $\beta$. Moreover, these functions are linear with respect to $\alpha$ and $\beta$. So, similar to the previous case, it is sufficient to investigate the signs of $G(u, v ; 0,0)$ and $G(u, v ; 1,-1)$. Here, we have

$$
\begin{aligned}
& G(u, v ; 0,0)=1-u v \geq 0, \quad u, v \in[0,1] \\
& G(u, v ; 1,-1) \\
& =1+(1-u)(1-v)-(1-u)^{2}(1-v)^{2}+u v[1-4(1-u) \\
& \quad \times(1-v)]-(u+v-2 u v)[1-2(1-u)(1-v)]-u v \\
& \quad-u v(1-u)(1-v)+u v(1-u)^{2}(1-v)^{2} \geq 0, \quad u, v \in[0,1] .
\end{aligned}
$$

This is illustrated by Figure 6(b). Then, we conclude that $g(u, v ; \alpha, \beta) \geq 0$, for $\alpha \in$ $[0,1]$ and $\beta \in[-1,1]$. Moreover, Figure 7 also illustrates that $G(u, v ; \alpha, \beta) \geq 0$ for certain values of $\alpha$ and $\beta$.

It is also noted that, based on Theorem 2.2(ii), the bivariate functions

$$
\begin{gathered}
C(u, v ; \alpha, \theta)=u v\left[1+\alpha\left(u^{2}-1\right)\left(v^{2}-1\right)\right] e^{\theta(1-u)(1-v)}, \\
0 \leq \alpha \leq 0.45,-1 \leq \theta \leq 0
\end{gathered}
$$

and

$$
\begin{gathered}
C(u, v ; \delta, \theta)=u v\left[1+\delta\left(1-u^{0.5}\right)\left(1-v^{0.5}\right)\right] e^{\theta(1-u)(1-v)}, \\
0 \leq \delta \leq 2,-1 \leq \theta \leq 0
\end{gathered}
$$

are copulas. 

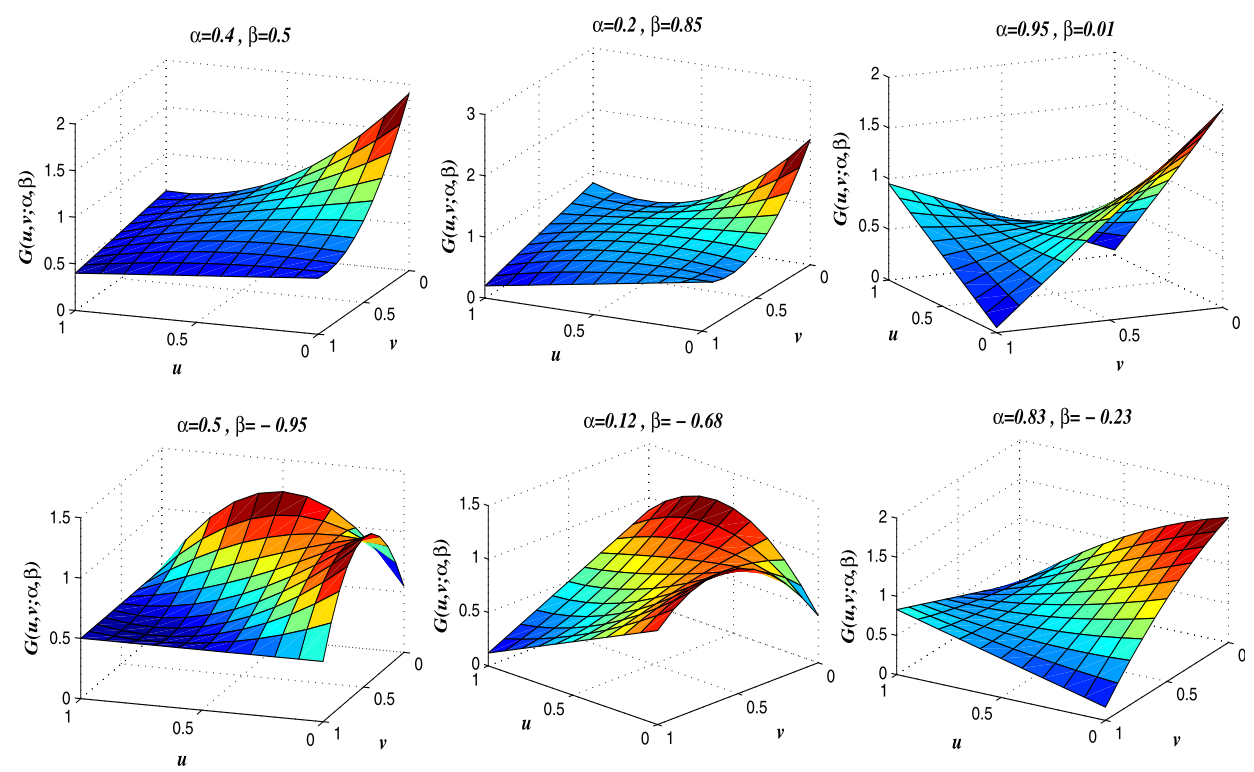

Figure 7 Illustrations of $G(u, v ; \alpha, \beta)$.

Remark 3.1. An important feature of the family introduced here is that it is very large so that it contains many copulas, several but not all Archimedean copulas included. For instance, by our Theorems 2.1 and 2.2 it follows that

$$
\begin{gathered}
C(u, v ; \delta, \theta, \gamma)=u v\left[1+\delta(1-u)^{2}(1-v)^{2}\right][1+\theta(1-u)(1-v)]^{\frac{1}{\gamma}} \\
0 \leq \delta \leq 1, \quad 0 \leq \theta \leq 1, \quad \gamma \geq 1, \quad \gamma \leq-1, \\
0 \leq \delta \leq 1, \quad-1 \leq \theta \leq 1, \quad \gamma=1, \\
\delta=0, \quad-1 \leq \theta \leq 1, \quad \gamma \leq-1,
\end{gathered}
$$

is a bivariate copula which is not Archimrdean. However, in the special case when $\delta=0$ and $\gamma=1$ it reduces to $F G M$ (the first order approximation to the Frank copula, which is Archimedean) and when $\delta=0$ and $\gamma=-1$ it reduces to the Archimedean copula $A M H$. Also, by Theorem 2.2, the bivariate function

$$
\begin{gathered}
C(u, v ; \delta, \theta)=u v e^{\left[(-\ln (u))^{-\delta}+(-\ln (v))^{-\delta}\right]^{\frac{-1}{\delta}}} e^{\theta \ln (u) \ln (v),}, \\
0<\delta \leq 1, \quad 0 \leq \theta \leq 1
\end{gathered}
$$

is a bivariate copula but it is not Archimrdean in general. In the special case when $\theta=0$, it reduces to Galambos copula and when $\delta \rightarrow 0$ reduces to $B G$ copula which is an Archimedean copula. 
Remark 3.2. Note that when $\phi$ is unbounded, such as $\ln (u)$, for any values of $\gamma$

$$
R(u, v ; \gamma)=\phi(u)+\frac{\phi^{\prime}(u)}{\gamma \frac{\partial}{\partial u} \ln K(u, v)} \quad \forall u, v \in I
$$

does not lie between $[-1,1]$ and thus $G$ is empty. Even if $\phi$ is bounded, $\phi^{\prime}$ may be unbounded such as

$$
\phi(u)=(1-u)^{k}, \quad 0<k<1
$$

in which case again $G$ is empty. As another instance, let $\phi$ and $\phi^{\prime}$ be bounded, e.g., $\phi=1-u$, but $K$ be the Clayton copula

$$
K(u, v ; \theta)=\left(u^{-\theta}+v^{-\theta}\right)^{\frac{-1}{\theta}} .
$$

Here, we have

$$
R(u, v ; \theta, \gamma)=1-u-\frac{u^{\theta+1}\left(u^{-\theta}+v^{-\theta}\right)}{\gamma},
$$

which can be unbounded for $v=0$ and thus $G$ is empty.

Similarly, for $Q_{0}, Q_{1}$ and $Q_{2}$ to be empty, one can consider $\phi(u)=1-u$ and $K$ as the Galambos copula.

\section{Dependence properties}

In this section, we shall investigate dependence properties of our new class of copulas. We shall show that, under certain conditions, dependent properties of $C(u, v)$ are the same as those of $K(u, v)$. First, we need to recall some known concepts of dependencies.

Definition 4.1. Let r.v.'s $X$ and $Y$ be related by a copula $C(u, v)$. Then,

1. $X$ and $Y$ are Positively Quadrant Dependent $(P Q D)$ (Negatively Quadrant Dependent $(N Q D))$, if

$$
\forall(x, y) \in \mathfrak{R}^{2}, \quad P(X \leq x, Y \leq y) \geq(\leq) P(X \leq x) P(Y \leq y)
$$

or, equivalently,

$$
\forall(u, v) \in I^{2}, \quad C(u, v) \geq(\leq) u v .
$$

2. $Y$ is Left Tail Decreasing (LTD) in $X$ if $P(Y \leq y \mid X \leq x)$ is nonincreasing in $x$ for all $y$.

3. $X$ and $Y$ are Left Corner Set Decreasing (LCSD) if $P(X \leq x, Y \leq y \mid X \leq$ $\left.x^{\prime}, Y \leq y^{\prime}\right)$ is nonincreasing in $x^{\prime}$ and $y^{\prime}$ for all $x$ and $y$.

4. $Y$ is Stochastically Increasing $(S I)$ in $X$ if $P(Y>y \mid X=x)$ is nondecreasing in $x$ for all $y$. 
Theorem 4.1. Let either $\forall u \in I, \phi(u) \geq 0$ or $\forall u \in I, \phi(u) \leq 0$.

(i) If $\theta \gamma \geq 0(\theta \gamma \leq 0)$, then $P Q D(N Q D)$ property of $K(u, v)$ is preserved by $C(u, v ; \theta, \gamma)=K(u, v)[1+\theta \phi(u) \phi(v)]^{\frac{1}{\gamma}}$.

(ii) If $\theta \in[0,1](\theta \in[-1,0])$, then $P Q D(N Q D)$ property of $K(u, v)$ is preserved by $C(u, v ; \theta)=K(u, v) e^{\theta \phi(u) \phi(v)}$.

Proof. (i) Suppose that $\gamma>0$ and $0 \leq \theta \leq 1$, then

$$
1+\theta \phi(u) \phi(v) \geq 1 \quad \forall u, v \in I,
$$

and, thus,

$$
[1+\theta \phi(u) \phi(v)]^{\frac{1}{\gamma}} \geq 1 \quad \forall u, v \in I .
$$

Hence, if $K(u, v)$ is $P Q D$, it follows that

$$
C(u, v ; \theta, \gamma)=K(u, v)[1+\theta \phi(u) \phi(v)]^{\frac{1}{\gamma}} \geq u v \quad \forall u, v \in I,
$$

as required. For $\gamma<0$ and $-1 \leq \theta \leq 0$, we have

$$
0 \leq 1+\theta \phi(u) \phi(v) \leq 1,
$$

or, equivalently,

$$
[1+\theta \phi(u) \phi(v)]^{\frac{1}{\gamma}} \geq 1 .
$$

Thus, if $K(u, v)$ is $P Q D$, it follows that

$$
K(u, v)[1+\theta \phi(u) \phi(v)]^{\frac{1}{\gamma}} \geq u v \quad \forall u, v \in I,
$$

as required. For $\gamma<0$ and $0 \leq \theta \leq 1$, we have

$$
1+\theta \phi(u) \phi(v) \geq 1,
$$

or, equivalently,

$$
[1+\theta \phi(u) \phi(v)]^{\frac{1}{\gamma}} \leq 1 .
$$

If $K(u, v)$ is $N Q D$, it follows that

$$
K(u, v)[1+\theta \phi(u) \phi(v)]^{\frac{1}{\gamma}} \leq u v \quad \forall u, v \in I,
$$

as required. For $\gamma>0$ and $-1 \leq \theta \leq 0$, we have

$$
0 \leq 1+\theta \phi(u) \phi(v) \leq 1 .
$$

Thus, if $K(u, v)$ is $N Q D$, we have

$$
K(u, v)[1+\theta \phi(u) \phi(v)]^{\frac{1}{\gamma}} \leq u v \quad \forall u, v \in I .
$$

i.e. $C(u, v ; \theta, \gamma)$ is $N Q D$. 
(ii) For $\theta \in[0,1](\theta \in[-1,0]), e^{\theta \phi(u) \phi(v)} \geq 1(\leq 1)$. Thus, if $K(u, v)$ is $P Q D(N Q D), C(u, v ; \theta)$ is also $P Q D(N Q D)$.

First, in what follow assume that $X$ and $Y$ are related according to copula $C(u, v ; \theta, \gamma)=K(u, v)[1+\theta \phi(u) \phi(v)]^{\frac{1}{\gamma}}$ and $X^{\prime}$ and $Y^{\prime}$ are related according to the corresponding base copula $K$.

Theorem 4.2. Let $\phi:[0,1] \rightarrow[-1,1]$ be a differentiable function and $\theta \gamma>0$. Then

(i) $Y$ is LTD $X$ if $Y^{\prime}$ is LTD in $X^{\prime}$ and $\phi$ is positive and nonincreasing or negative and nondecreasing.

(ii) $X$ and $Y$ are LCSD if $X^{\prime}$ and $Y^{\prime}$ are LCSD and $\phi$ is positive and nonincreasing or negative and nondecreasing.

(iii) $Y$ is $S I$ in $X$ if $Y^{\prime}$ is $S I$ in $X^{\prime}$ and $\phi$ is positive, nonincreasing and concave or negative, nondecreasing and convex for $0 \leq \theta \leq 1$ and $\gamma \geq 1$.

Proof. (i) By Theorem 5.2.5 in Nelsen (2006), it is sufficient to prove that $\frac{C(u, v ; \theta, \gamma)}{u}$ is nonincreasing in $u$ for all $v \in I$. Since

$$
\frac{C(u, v ; \theta, \gamma)}{u}=\frac{K(u, v)}{u}[1+\theta \phi(u) \phi(v)]^{\frac{1}{\gamma}},
$$

according to the condition of the theorem, $\frac{K(u, v)}{u}$ is nonincreasing in $u$. Obviously $[1+\theta \phi(u) \phi(v)]^{\frac{1}{\gamma}}$ is also nonincreasing in $u$, because

$$
\frac{d}{d u}[1+\theta \phi(u) \phi(v)]^{\frac{1}{\gamma}}=\frac{\theta}{\gamma} \phi^{\prime}(u) \phi(v)[1+\theta \phi(u) \phi(v)]^{\frac{1}{\gamma}-1} \leq 0 .
$$

(ii) By Corollary 5.2.17 in Nelsen (2006), we should show that for all $0 \leq u_{1} \leq$ $u_{2} \leq 1$ and $0 \leq v_{1} \leq v_{2} \leq 1$,

$$
C\left(u_{1}, v_{1} ; \theta, \gamma\right) C\left(u_{2}, v_{2} ; \theta, \gamma\right)-C\left(u_{1}, v_{2} ; \theta, \gamma\right) C\left(u_{2}, v_{1} ; \theta, \gamma\right) \geq 0,
$$

or equivalently,

$$
\begin{aligned}
& K\left(u_{1}, v_{1}\right) K\left(u_{2}, v_{2}\right)\left\{\left[1+\theta \phi\left(u_{1}\right) \phi\left(v_{1}\right)\right]\left[1+\theta \phi\left(u_{2}\right) \phi\left(v_{2}\right)\right]\right\}^{\frac{1}{\gamma}} \\
& \quad-K\left(u_{1}, v_{2}\right) K\left(u_{2}, v_{1}\right)\left\{\left[1+\theta \phi\left(u_{1}\right) \phi\left(v_{2}\right)\right]\left[1+\theta \phi\left(u_{1}\right) \phi\left(v_{2}\right)\right]\right\}^{\frac{1}{\gamma}} \geq 0 .
\end{aligned}
$$

First, suppose that $0 \leq \theta \leq 1$ and $\gamma \geq 0$. Since $X^{\prime}$ and $Y^{\prime}$ are $L C S D$, it is sufficient to show that

$$
\begin{aligned}
\left\{\left[1+\theta \phi\left(u_{1}\right) \phi\left(v_{1}\right)\right]\left[1+\theta \phi\left(u_{2}\right) \phi\left(v_{2}\right)\right]\right\}^{\frac{1}{\gamma}} \geq & \left\{\left[1+\theta \phi\left(u_{1}\right) \phi\left(v_{2}\right)\right]\right. \\
& \left.\times\left[1+\theta \phi\left(u_{2}\right) \phi\left(v_{1}\right)\right]\right\}^{\frac{1}{\gamma}}
\end{aligned}
$$


or, equivalently,

$$
\left[\phi\left(v_{2}\right)-\phi\left(v_{1}\right)\right]\left[\phi\left(u_{2}\right)-\phi\left(u_{1}\right)\right] \geq 0 .
$$

But, by monotonicity of $\phi,(4.5)$ obviously holds and, thus, we have the result. By the same argument, (4.4) holds for $-1 \leq \theta \leq 0$ and $\gamma \leq 0$.

(iii) By Corollary 5.2.11 in Nelsen (2006) we should show that $\frac{\partial C(u, v)}{\partial u}$ is nonincreasing in $u$. But, we have

$$
\begin{aligned}
\frac{\partial C(u, v ; \theta, \gamma)}{\partial u}= & \frac{\partial}{\partial u}\left\{K(u, v)[1+\theta \phi(u) \phi(v)]^{\frac{1}{\gamma}}\right\} \\
= & \frac{\partial K(u, v)}{\partial u} \underbrace{[1+\theta \phi(u) \phi(v)]^{\frac{1}{\gamma}}}_{I} \\
& +\frac{K(u, v)}{u} \underbrace{\left\{\frac{\theta}{\gamma} u \phi(v) \phi^{\prime}(u)[1+\theta \phi(u) \phi(v)]^{\frac{1}{\gamma}-1}\right\}}_{I I} .
\end{aligned}
$$

Since $Y^{\prime}$ is $S I$ in $X^{\prime}$ and $S I$ implies LTD (Corollary 5.2.17 in Nelsen, 2006), $\frac{\partial K(u, v)}{\partial u}$ and $\frac{K(u, v)}{u}$ are nonincreasing. Expressions I and II are also nonincreasing because

$$
\frac{\partial}{\partial u}[1+\theta \phi(u) \phi(v)]^{\frac{1}{\gamma}}=\frac{\theta}{\gamma} \phi^{\prime}(u) \phi(v)[1+\theta \phi(u) \phi(v)]^{\frac{1}{\gamma}-1} \leq 0
$$

and

$$
\begin{aligned}
& \frac{\partial}{\partial u}\left\{\frac{\theta}{\gamma} u \phi^{\prime}(u) \phi(v)[1+\theta \phi(u) \phi(v)]^{\frac{1}{\gamma}-1}\right\} \\
& =\frac{\theta}{\gamma} \phi^{\prime}(u) \phi(v)[1+\theta \phi(u) \phi(v)]^{\frac{1}{\gamma}-1} \\
& \quad+\frac{\theta}{\gamma} \phi^{\prime \prime}(u) u \phi(v)[1+\theta \phi(u) \phi(v)]^{\frac{1}{\gamma}-1}+\frac{\theta^{2}}{\gamma^{2}}(1-\gamma)\left(\phi^{\prime}(u)\right)^{2}(\phi(v))^{2} u \\
& \quad \times[1+\theta \phi(u) \phi(v)]^{\frac{1}{\gamma}-2} \leq 0
\end{aligned}
$$

Consequently, by (4.8) and (4.9), the Expressions (4.6) and (4.7) are nonincreasing in $u$. This completes the proof.

Now, in what follows, we assume that $X$ and $Y$ are related according to copula $C(u, v ; \theta)=K(u, v) e^{\theta \phi(u) \phi(v)}$ and $X^{\prime}$ and $Y^{\prime}$ are related according to the corresponding base copula $K$.

Theorem 4.3. Let $\phi$ be a differentiable function and $0 \leq \theta \leq 1$. Then 
(i) $Y$ is LTD in $X$ if $Y^{\prime}$ is LTD in $X^{\prime}$ and $\phi$ is positive and nonincreasing or negative and nondecreasing.

(ii) $Y$ is LCSD in $X$ if $Y^{\prime}$ is LCSD in $X^{\prime}$ and $\phi$ is positive and nonincreasing or negative and nondecreasing.

Proof. (i) It is sufficient to prove that $\frac{C(u, v ; \theta)}{u}$ is nonincreasing in $u$. According to the condition of the theorem, $\frac{K(u, v)}{u}$ is nonincreasing in $u$ and obviously $e^{\theta \phi(u) \phi(v)}$ is nonincreasing in $u$ too, because we have

$$
\frac{\partial}{\partial u} e^{\theta \phi(u) \phi(v)}=\theta \phi^{\prime}(u) \phi(v) e^{\theta \phi(u) \phi(v)} \leq 0 .
$$

Hence,

$$
\frac{C(u, v ; \theta)}{u}=\frac{K(u, v)}{u} e^{\theta \phi(u) \phi(v)}
$$

is nonincreasing.

(ii) Similar to the proof of Theorem 4.2(ii).

\section{Tail dependence}

In this section, we consider two concepts of dependencies that measure the amount of dependency in the upper-right quadrant and lower-right quadrant on $I^{2}$ and show that upper tail dependence coefficient of $(X, Y)$ equals to that of $\left(X^{\prime}, Y^{\prime}\right)$.

Definition 5.1. Let $X$ and $Y$ be continuous random variables with d.f.'s $F$ and $G$, respectively. The upper tail dependence $\lambda_{U}$ is defined by

$$
\lambda_{U}=\lim _{u \rightarrow 1^{-}} P\left[Y>G^{-1}(u) \mid X>F^{-1}(u)\right]=2-\lim _{u \rightarrow 1^{-}} \frac{1-C(u, u)}{1-u} .
$$

Similarly, lower tail dependence $\lambda_{L}$ is defined by

$$
\lambda_{L}=\lim _{u \rightarrow 0^{+}} P\left[Y \leq G^{-1}(u) \mid X \leq F^{-1}(u)\right]=\lim _{u \rightarrow 0^{+}} \frac{C(u, u)}{u} .
$$

In the following theorem, we assume that $\lambda_{U, K}$ and $\lambda_{L, K}$ are upper and lower tail dependencies of copula $K(u, v)$, and $\lambda_{U, C}$ and $\lambda_{L, C}$ are those of copula $C(u, v ; \theta, \gamma)($ or $C(u, v ; \theta))$.

\section{Theorem 5.1.}

(i) If $C(u, v ; \theta, \gamma)=K(u, v)[1+\theta \phi(u) \phi(v)]^{\frac{1}{\gamma}}$ for $\gamma \neq 0$, then

$$
\lambda_{U, C}=\lambda_{U, K}, \quad \lambda_{L, C}=\lambda_{L, K}[1+\theta \phi(0)]^{\frac{1}{\gamma}} .
$$


(ii) if $C(u, v ; \theta)=K(u, v) e^{\theta \phi(u) \phi(v)}$, then

$$
\lambda_{U, C}=\lambda_{U, K}, \quad \lambda_{L, C}=\lambda_{L, K} e^{\theta \phi^{2}(0)} .
$$

Proof. (i) We have

$$
\begin{aligned}
\lambda_{U, C}= & 2-\lim _{u \rightarrow 1^{-}} \frac{1-C(u, u)}{1-u} \\
= & 2-\lim _{u \rightarrow 1^{-}} \frac{1-K(u, u)\left[1+\theta \phi^{2}(u)\right]^{\frac{1}{\gamma}}}{1-u} \\
= & 2-\lim _{u \rightarrow 1^{-}}\left\{\frac{\partial}{\partial u} K(u, u)\left[1+\theta \phi^{2}(u)\right]^{\frac{1}{\gamma}}+\frac{2 \theta}{\gamma} K(u, u) \phi(u) \phi^{\prime}(u)\right. \\
& \left.\times\left[1+\theta \phi^{2}(u)\right]^{\frac{1}{\gamma}-1}\right\} \\
= & 2-\lim _{u \rightarrow 1^{-}} \frac{\partial}{\partial u} K(u, u)=\lambda_{U, K}
\end{aligned}
$$

and

$$
\begin{aligned}
\lambda_{L, C}= & \lim _{u \rightarrow 0^{+}} \frac{C(u, u)}{u} \\
= & \lim _{u \rightarrow 0^{+}} \frac{\partial}{\partial u} C(u, u) \\
= & \lim _{u \rightarrow 0^{+}}\left\{\frac{\partial}{\partial u} K(u, u)\left[1+\theta \phi^{2}(u)\right]^{\frac{1}{\gamma}}+\frac{2 \theta}{\gamma} K(u, u) \phi(u) \phi^{\prime}(v)\right. \\
& \left.\times\left[1+\theta \phi^{2}(u)\right]^{\frac{1}{\gamma}-1}\right\} \\
= & \left(\lim _{u \rightarrow 0^{+}} \frac{\partial}{\partial u} K(u, u)\right)\left[1+\theta \phi^{2}(0)\right]^{\frac{1}{\gamma}} \\
= & \lambda_{L, K}\left[1+\theta \phi^{2}(0)\right]^{\frac{1}{\gamma}} .
\end{aligned}
$$

As required. The prove of part (ii) is similar.

\section{Measures of association}

In this section, we obtain Spearman's $\rho_{s}$ for our new copulas for certain values of their parameters. As theoretical calculations are not feasible here, we shall use a Maple software to compute $\rho_{s}$ numerically. Our new copulas prove to improve the range of correlation coefficient of the FGM family. Recall that Spearman's $\rho_{s}$ is 
Table 2 Spearman's $\rho_{s}$ for various values of $k, \delta, \theta$ and $\gamma$ for copula (3.1)

\begin{tabular}{lllllllllc}
\hline$k$ & $\delta$ & $\theta$ & $\gamma$ & $\rho_{S}$ & $k$ & $\delta$ & $\theta$ & $\gamma$ & $\rho_{S}$ \\
\hline 1 & 1 & 1 & 1 & 0.4467 & 1.5 & 1 & -1 & -4 & 0.2972 \\
3 & 2 & 1 & 3 & 0.5116 & 2 & 1 & -1 & -6 & 0.2945 \\
3 & 0.5 & 0.75 & 4 & 0.2241 & 0 & 1 & -1 & -2 & 0.0833 \\
2.4 & 2 & 1 & 3 & 0.4588 & 1 & 1 & -1 & -2 & 0.3230 \\
1 & 2 & 0 & 3 & 0.1667 & 1 & 1 & -1 & -4 & 0.1969 \\
0.5 & 1 & 1 & 1 & 0.2160 & 2 & 1 & -1 & -4 & 0.4143 \\
3 & 1 & -1 & -6 & 0.4597 & 2.5 & 1 & -1 & -6 & 0.4847 \\
\hline
\end{tabular}

Table 3 The Spearman's $\rho_{s}$ for various values of $k, \alpha, \theta$ and $\gamma$ for copula (3.2)

\begin{tabular}{lccccccrrc}
\hline$k$ & $\delta$ & $\theta$ & $\gamma$ & $\rho_{s}$ & $k$ & $\delta$ & $\theta$ & $\gamma$ & $\rho_{S}$ \\
\hline 0.1 & 0.4 & 1 & 0.5 & 0.3167 & 0.2 & 0.001 & 1 & 1 & 0.0256 \\
0.5 & 0.4 & 1 & 2.5 & 0.3561 & 1 & 0.4 & -1 & -5 & 0.3981 \\
1 & 0.4 & 1 & 5 & 0.3728 & 2 & 0.4 & -1 & -10 & 0.4339 \\
1 & 0.4 & 1 & 6 & 0.3604 & 3 & 0.4 & -1 & -15 & 0.4516 \\
3 & 0.4 & 1 & 15 & 0.3677 & 3 & 0.4 & 0 & -10 & 0.3000 \\
1 & 0.01 & 1 & 5 & 0.0689 & 5 & 0.4 & -1 & -25 & 0.4681 \\
1 & 0.25 & 1 & 5 & 0.2559 & 1 & 0.4 & -1 & -8 & 0.3604 \\
0.75 & 0.4 & 1 & 3.75 & 0.3671 & 1 & 0.4 & -1 & -7 & 0.3693 \\
6 & 0.4 & -1 & -30 & 0.4723 & 4 & 0.4 & -1 & -20 & 0.4618 \\
\hline
\end{tabular}

defined by

$$
\rho_{s}=12 \int_{0}^{1} \int_{0}^{1} C(u, v) d u d v-3
$$

\section{Example 6.1.}

- 6.1.1. Consider the copula in (3.1). The Spearman's $\rho_{s}$ calculated for various values of $k, \delta, \theta$ and $\gamma$ appear in Table 2. Here, $\rho_{s}$ is an increasing function in $\delta$ and $k$, increasing (decreasing) function in $\theta$ when $\gamma \geq 0 \gamma \leq 0$ ) and increasing (decreasing) function in $\gamma$ when $\theta \leq 0(\theta \geq 0)$. Furthermore, by Theorem 4.1, $C(u, v ; k, \delta, \theta, \gamma)$ is $P Q D$. So, $\rho_{s} \in[0,0.5116]$.

- 6.1.2. Consider the copula in (3.2). $\rho_{s}$ is increasing in $\alpha$ and $k$, increasing (decreasing) function in $\theta$ if $\gamma \geq 0(\gamma \leq 0)$, increasing (decreasing) function in $\gamma$ if $\theta \leq 0(\theta \geq 0)$. The Spearman's $\rho_{s}$ calculated for various values of its parameters appear in Table 3. The value of $\rho_{s}$ equals 0.4723 for $k=6, \alpha=0.4, \theta=-1$ and $\gamma=-30$ which is greater than the upper bound of the correlation coefficient of an FGM copula.

- 6.1.3. Consider the copula in (3.3). The Spearman's $\rho_{s}$ calculated for various values of $\delta, \theta, \gamma$ and $p$ appear in Table 4. Here, $\rho_{s}$ is increasing function in $p$ 
Table 4 The Spearman's $\rho_{s}$ for various values of $\delta, \theta, \gamma$ and $p$ for copula (3.3)

\begin{tabular}{llllcccccc}
\hline$\delta$ & $\theta$ & $\gamma$ & $p$ & $\rho_{S}$ & $\delta$ & $\theta$ & $\gamma$ & $p$ & $\rho_{S}$ \\
\hline 5 & 1 & 2 & 0.1 & 0.1964 & 0.1 & -1 & -2 & 5 & 0.3857 \\
0.5 & 1 & 2 & 1 & 0.3437 & 0.5 & -1 & -2 & 1 & 0.4122 \\
0.25 & 1 & 2 & 2 & 0.3643 & 0.25 & -1 & -2 & 2 & 0.4311 \\
0.1 & 1 & 2 & 5 & 0.3233 & 5 & -1 & -2 & 0.1 & 0.2571 \\
1.67 & 1 & 2 & 0.3 & 0.2542 & 0.5 & -1 & -3 & 1 & 0.3248 \\
0.25 & 1 & 2 & 2 & 0.3643 & 0.05 & -0.5 & -3 & 6 & 0.1480 \\
0.17 & 0.5 & 2 & 3 & 0.2733 & 5 & 0 & -2 & 0.1 & 0.0340 \\
0 & 1 & 3 & 1 & 0.1033 & 0.5 & 0 & 3 & 1 & 0.1667 \\
\hline
\end{tabular}

Table 5 The Spearman's $\rho_{s}$ for various values of $\alpha, \theta, \beta$ and $\gamma$ for copula (3.4)

\begin{tabular}{llllcllllc}
\hline$\alpha$ & $\theta$ & $\beta$ & $\gamma$ & $\rho_{s}$ & $\alpha$ & $\theta$ & \multicolumn{1}{c}{$\beta$} & \multicolumn{1}{c}{$\gamma$} & $\rho_{s}$ \\
\hline 0.1 & -0.35 & 0.5 & -3 & 0.0849 & 0 & 1 & 1 & 1.5 & 0.1397 \\
0.2 & -0.75 & 0.5 & -1 & 0.1769 & 0.2 & 0.5 & 0.9 & 1 & 0.1862 \\
0.2 & -1 & 1 & -1 & 0.2484 & 0.5 & 1 & 1 & 1.5 & 0.3134 \\
0.3 & -0.95 & 0.8 & -2 & 0.2124 & 0.5 & 1 & 1 & 2 & 0.2973 \\
0.45 & -0.5 & 1 & -1.5 & 0.2663 & 0.6 & 0.85 & 0.8 & 1.23 & 0.3332 \\
0.65 & -1 & 1 & -2.5 & 0.3425 & 0.8 & 1 & 1 & 4 & 0.3750 \\
0.8 & -1 & 0 & -4 & 0.2916 & 0.95 & 1 & 0.7 & 14.72 & 0.3818 \\
0.99 & -1 & 1 & -75 & 0.4148 & 0.99 & 0 & 1 & -75 & 0.4133 \\
0.99 & -1 & 1 & -73.58 & 0.4149 & 0.99 & 1 & 1 & 73.58 & 0.4147 \\
\hline
\end{tabular}

and $\delta$, increasing (decreasing) function in $\theta$ when $\gamma \geq 0(\gamma \leq 0)$ and increasing (decreasing) function in $\gamma$ when $\theta \leq 0(\theta \geq 0)$. The value of $\rho_{s}$ equals 0.4311 for $\delta=0.25, \theta=-1, \gamma=-2$ and $p=2$ which is greater than the upper bound of the correlation coefficient of an FGM copula.

- 6.1.4. Consider the copula in (3.4). $\rho_{s}$ is an increasing function in $\alpha$ and $\beta$, increasing (decreasing) function in $\theta$ when $\gamma \geq 0(\gamma \leq 0)$ and increasing (decreasing) function in $\gamma$ if $\theta \geq 0(\theta \leq 0)$. The Spearman's $\rho_{s}$ calculated for various values of its parameters appear in Table 5. The value of $\rho_{s}$ equals 0.4149 for $\alpha=0.99, \theta=-1, \beta=1$ and $\gamma=-73.58$ which is greater than the upper bound of the correlation coefficient of an FGM copula.

- 6.1.5. Consider the copula in (3.8). Table 6 shows Spearman's $\rho_{s}$ values calculated for various values of $\alpha, \beta$ and $\theta . \rho_{s}$ is an increasing function in $\alpha, \beta$ and $\theta$. Furthermore, $C(u, v ; \alpha, \beta, \theta)$ is $P Q D$, so $\rho_{s} \in[0,0.3515]$.

- 6.1.6. Consider the copula in (3.9). $\rho_{s}$ is increasing in $\alpha, \beta$ and $\theta$. As seen in Table 7, the maximum of $\rho_{s}$ equals 0.4167 which is greater than the upper bound of an FGM copula and the minimum of $\rho_{s}$ equals -0.3552 which is less than the lower bound of an FGM copula. 
Table 6 The Spearman's $\rho_{s}$ for various values of parameters of copula (3.8)

\begin{tabular}{llcccccc}
\hline$\alpha$ & $\beta$ & $\theta$ & $\rho_{S}$ & $\alpha$ & $\beta$ & $\theta$ & $\rho_{S}$ \\
\hline 0 & 0 & 0.1 & 0.0078 & 0.45 & 0.3 & 0.6 & 0.2300 \\
0.1 & 0.1 & 0.2 & 0.0579 & 0.5 & 0.35 & 0.7 & 0.2614 \\
0.2 & 0.15 & 0.3 & 0.1043 & 0.55 & 0.4 & 0.8 & 0.2931 \\
0.3 & 0.2 & 0.4 & 0.1514 & 0.6 & 0.45 & 0.9 & 0.3253 \\
0.4 & 0.25 & 0.5 & 0.1991 & $1-e^{-1}$ & 0.5 & 1 & 0.3515 \\
\hline
\end{tabular}

Table 7 The Spearman's $\rho_{s}$ for various values of parameters of copulas (3.9) and (3.10)

\begin{tabular}{|c|c|c|c|c|c|c|}
\hline \multicolumn{4}{|c|}{ (3.9) } & \multicolumn{3}{|c|}{$(3.10)$} \\
\hline$\alpha$ & $\beta$ & $\theta$ & $\rho_{s}$ & $\delta$ & $\theta$ & $\rho_{s}$ \\
\hline 0.1 & -0.45 & -0.5 & -0.1588 & 0 & -1 & -0.2962 \\
\hline 0.4 & -.65 & -0.8 & -0.1733 & 0.1 & -0.9 & -0.2073 \\
\hline 0.8 & -0.95 & -1 & -0.1418 & 0.15 & -0.8 & -0.1471 \\
\hline 0.8 & -0.95 & 0 & 0.1875 & 0.2 & -0.7 & -0.0850 \\
\hline 1 & -1 & -1 & -0.0920 & 0.25 & -0.6 & -0.0208 \\
\hline 0 & -1 & -1 & -0.3552 & 0.3 & -0.5 & 0.0455 \\
\hline 0.6 & 0.2 & -0.35 & 0.0865 & 0.35 & -0.4 & 0.1141 \\
\hline 0.9 & 0.8 & -0.7 & 0.0911 & 0.4 & -0.3 & 0.1849 \\
\hline 1 & 1 & -1 & 0.0258 & 0.45 & -0.2 & 0.2582 \\
\hline 1 & 1 & 0 & 0.4167 & 0.45 & 0 & 0.3375 \\
\hline
\end{tabular}

- 6.1.7. Consider the copula in (3.10). $\rho_{s}$ is an increasing in $\delta$ and $\theta$. Hence, as we see in Table $7, \rho_{s} \in[-0.2962,0.3375]$.

Remark 6.1. For copula (3.12) which contains $F G M$ and $A M H, \rho_{s} \in[-0.28$, $0.4784]$.

\section{Conclusion}

In this study, we started with a rather arbitrary base bivariate copula and found sufficient and necessary conditions under which two new classes of bivariate functions would be copulas. In particular, they include various already known copulas previously studied in the literature such as FGM, AMH and BG. Dependence properties of variables involved were investigated and revealed that the dependent properties of the base copula are preserved by the generated copula under certain conditions. Furthermore, we have provided several examples and computed the values of Spearman's $\rho_{s}$ as measures of association. It is shown that the proposed copulas improve this range of $\rho_{s}$ comparing to FGM copula. 


\title{
Acknowledgments
}

Authors are grateful to two anonymous reviewers for their constructive comments. The first author is also grateful to the Graduate Office of the University of Isfahan for their support.

\section{References}

Amblard, C. and Girard, S. (2002). Symmetry and dependence properties within a semiparametric family of bivariate copulas. Nonparametric Statistics 14, 715-727. MR1941711

Amblard, C. and Girard, S. (2009). A new extension of bivariate FGM copulas. Metrika 70, 1-17. MR2506497

Amini, M., Jabbari, H. and Mohtashami Borzadaran, G. R. (2011). Aspects of dependence in generalized Farlie-Gumbel-Morgenstern distribution. Communications in Statistics-Simulation and Computation 40, 1192-1205. MR2818099

Bairamov, I. and Kotz, S. (2002). Dependence structure and symmetry of Huang-Kotz FGM distributions and their extensions. Metrika 56, 55-72.

Cook, R. D. and Johnson, M. E. (1986). Generalized Burr-Pareto-logistic distributions with applications to a uranium exploration data set. Technometrics 28, 123-131. MR0841485

Delahorra, J. and Fernandez, C. (1995). Sensitivity to prior independence via Farlie-GumbelMorgenstern model. Communications in Statistics-Theory Methods 24, 987-996. MR1323259

Grane, G. J. (2009). Copulas for credit derviative pricing and other application. Ph.D. thesis.

Huang, J. S. and Kotz, S. (1999). Modifications of the Farlie-Gumbel-Morgenstern distribution. A tough hill to climb. Metrika 49, 135-145.

Klein, I. and Christa, F. (2011). Families of copulas closed under the construction of generalized linear means. IWQW discussion paper series, No. 04/2011.

Lin, G. D. (1987). Relationships between two extensions of Farlie-Gumbel-Morgenstern distribution. Annals of the Institute of Statistical Mathematics 39, 129-140. MR0886511

Nelsen, R. B. (2006). An Introduction to Copulas. New York: Springer. MR2197664

Sklar, A. (1959). Functions de repartition a n dimensions et leurs marges. Publication de Institut Statistique de Universite de Paris 8, 229-231. MR0125600

Tolley, H. D. and Norman, J. E. (1979). Time on trial estimates with bivariate risks. Biometrika 66, 285-291. MR0548195

\author{
Department of Statistics \\ University of Isfahan \\ Isfahan 81744 \\ Iran \\ E-mail: zahra.sharif@sci.ui.ac.ir \\ alamatho@sci.ui.ac.ir \\ i.kazemi@stat.ui.ac.ir
}

